\title{
Difficult decisions regarding percutaneous endoscopic gastrostomy placement
}

\author{
Guido Van Rosendaal MD FRCPC
}

$T^{h}$ he decision to place a percutaneous endoscopic gastrostomy (PEG) is easy when it is clear that long term nutritional support will result in a substantial improvement in the health of the recipient. Unfortunately, the endoscopist is regularly faced with requests for consideration of PEG insertion when the anticipated benefits are not so clear. The value of this intervention may be questionable for individuals with little hope of meaningful recovery.

Take, for example, the case of a 60-year-old man who was admitted to hospital a month previously with a massive stroke. He has a major neurological deficit, reacting only to noxious stimuli. Nasogastric tube feeding was initiated early in his course in an automatic fashion; no active decision making regarding nutritional support occurred at this stage. Is placement of a PEG in this man's best interest? Who makes the decision and what influences affect this decision? What is the endoscopist's role in this process of decision making? Should he or she assume that due consideration has been given to the relevant issues and simply provide a technical service? Or is there a requirement for active and comprehensive discussion with his surrogate decision makers regarding the impact of PEG feeding on his future course? Is there really an alternative to PEG feeding? It is important for us to consider the process of deciding care for such patients.

A central tenet of our bioethics framework is that individuals are autonomous - they have the right to make decisions for themselves regarding the care that they will receive. When they are unable to make these decisions themselves, we rely on surrogates, usually close family members or legal guardians, to make these choices on their behalf. We expect that such surrogates will know what the patient would want in the circumstances encountered and that they would support these preferences. Unfortunately, for a wide range of life-threatening circumstances, such decision makers appear to be unable to predict the wishes of the persons for whom they are responsible (1-4). Moreover, when choices have been indicated in advance directives, these appear not to influence decisions that are made regarding life-sustaining care (5). We seem unable, therefore, to assure that patient wishes and their right to autonomy are realized when they become unable to express their personal desires for life-sustaining care.

Surrogates regularly make their decisions in a state of emotional distress with inadequate information regarding PEG devices, the method of their placement, the effect that PEG feeding will have on the clinical course or regarding the prognosis of the underlying disease (6). They may hope desperately for a miraculous recovery and be burdened by guilt at the prospect of being responsible for withdrawing life support from someone they love and for whom they are responsible. Their view of the likely course of the underlying illness is often overly optimistic, and an abhorrence of death by starvation is often expressed; the need to provide nourishment seems to be fundamental to close human relationships.

Surrogates regularly regret the decisions that they have made once they have personally observed the evolution of 
the underlying disease and the poor quality of life that may follow the institution of PEG feeding. In one study, $41 \%$ of such individuals said they would not make the same decision for themselves (7).

Decision makers regularly relate to a large number of health professionals who participate in the provision of care. Several services are often involved, and over an extended hospitalization, it is highly likely that more than one physician will represent each discipline, particularly when care is provided in a teaching hospital. There may be no family physician providing longitudinal care, and because of the way that hospital staffing has evolved, it is now less likely that the families of such patients will develop close relationships with individual nurses or other members of the hospital staff. The lack of a prolonged and close relationship with a health professional in whom decision makers can place their trust may also compromise the ability to make optimal decisions.

The role of the endoscopists must not be limited to that of technician in the placement of PEGs. She or he has a responsibility to ensure that due consideration has been given to all of the relevant issues and options available, and that the choice made is the one that best coincides with the wishes of the person for whom the decision is being made.

What, then, can we do to optimize decisions to commit to long term nutritional support via PEG when these decisions are made on behalf of severely disabled individuals whose prognosis is bleak? The following recommendations warrant consideration.

- The person with legal decision-making authority must be identified and be fully informed regarding the procedure of PEG placement, its risks and possible complications, and the anticipated effect of feeding on the patient's future course. This must include a comprehensive discussion of the issues related to long term nutritional support via PEG with at least one physician.

- The prognosis of the underlying condition, particularly the quality of life to be anticipated, must be established as accurately as possible, ideally based on objective high quality evidence and/or expert opinion. This information should be presented to decision makers in an understandable and sensitive manner. We must continue to document experience with the outcomes of a wide range of disease conditions to facilitate fully informed decision making.

- There should be widespread encouragement at a societal level for all individuals to express their preference for life-supporting care before lifethreatening crises occur, particularly through advanced directives. These preferences, specified by directive or otherwise expressed, must be identified and respected in arriving at a decision regarding the placement of a PEG.
- When decision making is difficult, personal support should be provided. This may include personal counselling, use of specifically developed aids to decision making such as videotapes or access to support groups that include individuals who have had prior experience in similar decision-making. When conflicts arise in decision making, consultation with an ethics committee or another form of mediation should be offered.

- When the prognosis appears poor but aggressive supportive therapy is desired, a decision to undertake a time-limited trial of nutritional support should be considered before the insertion of a PEG. The goals of this intervention and the time period in which these goals are to be achieved should be clearly defined. If these goals are not achieved, the discontinuation of feeding is ethically acceptable (8). Reported experience indicates that the withholding of nutrition from dying patients does not increase their suffering.

Difficult decisions regarding life-sustaining interventions often invoke the concept of medical futility (9), the definition of which is likely to be the subject of vigorous ongoing debate. Beliefs in the 'sanctity of life' or life preservation regardless of quality, and/or at any cost because of religious beliefs, or ethnic or cultural values are likely to remain in a state of tension with concerns over the quality of life provided. When an underlying illness is devastating and a return to a meaningful quality of life is not possible, it may be more humane to allow the individual to succumb to their underlying disease than to provide for a life of such poor quality that the person living it would have declined the intervention, had they been able to make this decision for themselves.

\section{REFERENCES}

1. Hare J, Pratt C, Nelson C. Agreement between patients and their self-selected surrogates on difficult medical decisions. Arch Intern Med 1992;152:1049-54.

2. Layde PM, Beam CA, Broste SK, et al. Surrogates predictions of seriously ill patients' resuscitation preferences. Arch Fam Med 1995;4:518-23.

3. Suhl J, Reedy T, Garrick T. Myth of substituted judgement. Surrogate decision making regarding life support is unreliable. Arch Intern Med 1994;154:90-6.

4. Uhlmann RF, Pealman RA, Cain KC. Physicians' and spouses' predictions of elderly patients' resuscitation preferences. J Gastroenterol 1988;43:115-21.

5. Teno JM, Lynn J, Phillips RS, et al. Do formal advance directives affect resuscitation decisions and the use of resources for seriously ill patients? J Clin Ethics 1994;5:23-30.

6. Van Rosendaal G, Verhoef MJ, Kinsella TD. How are decisions made about the use of percutaneous endoscopic gastrostromy for long-term nutritional support? Am J Gastroenterol 1999;94:3225-8.

7. Verhoef MJ, Van Rosendaal GMA. Patient outcomes related to percutaneous endoscopic gastrostomy (PEG) placement. Am J Gastroenterol. (In press)

8. The Hastings Center. Guidelines on the Termination of Lifesustaining Treatment and the Care of the Dying. Bloomington: Indiana Press, 1987.

9. Pellegrino E. Decisions to withdraw life-sustaining treatment: A moral algorythm. JAMA 2000;283:1065-7. 


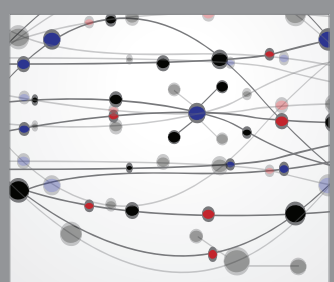

The Scientific World Journal
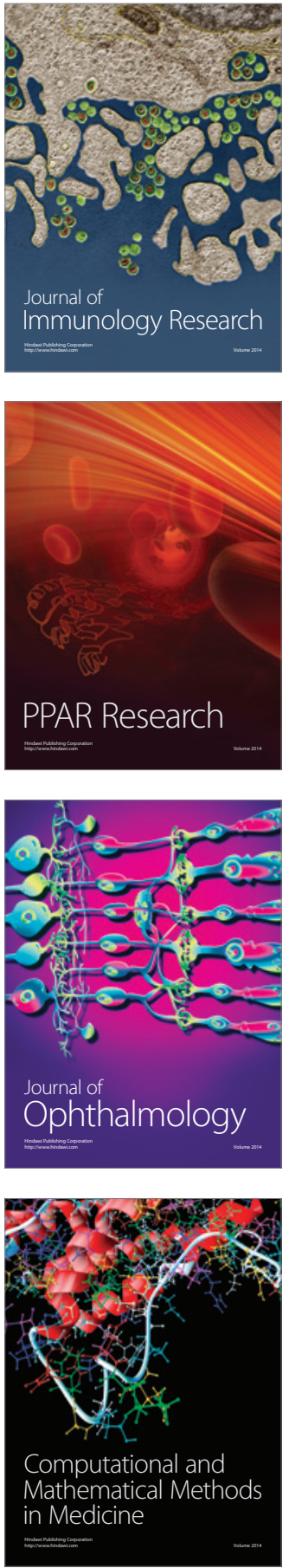

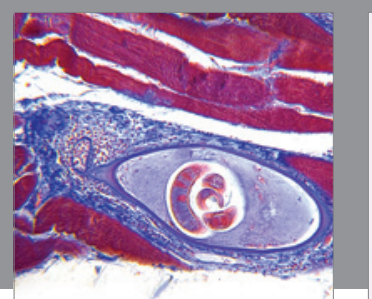

Gastroenterology Research and Practice

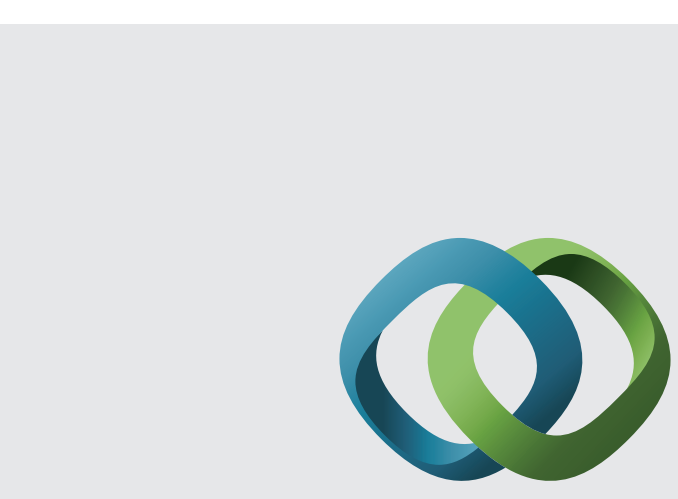

\section{Hindawi}

Submit your manuscripts at

http://www.hindawi.com
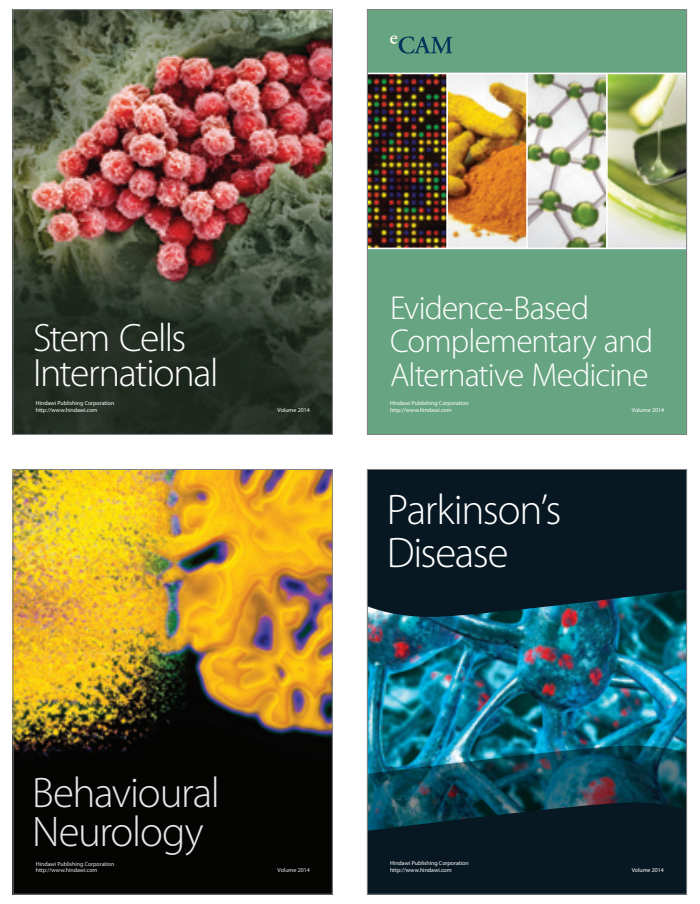
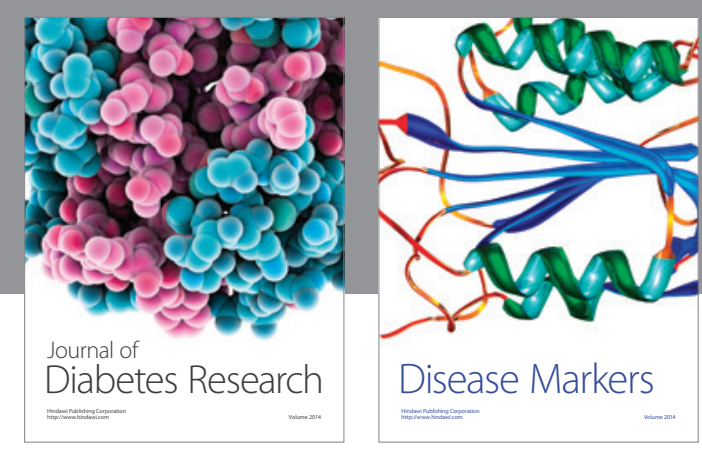

Disease Markers
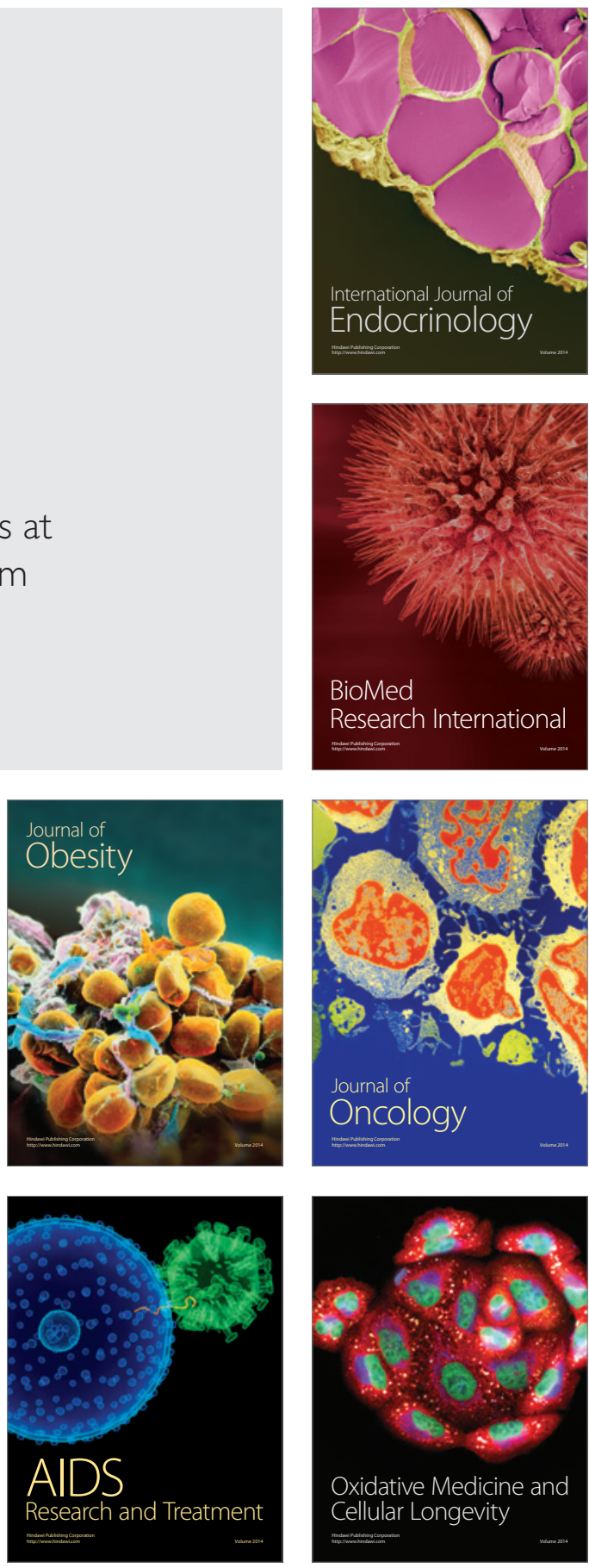